\title{
Blood Biochemical Reference Intervals for Free-Ranging Olive Baboons (Papio anubis) in Kenya
}

\author{
Mathew Mutinda ${ }^{1}$ - Margaret C. Crofoot ${ }^{2,3,4}$. Jennifer C. Kishbaugh ${ }^{5}$. \\ Lee-Ann C. Hayek ${ }^{6}$. Dawn Zimmerman ${ }^{5} \cdot$ Devin A. Tunseth $^{5} \cdot$ Suzan Murray $^{5}$
}

\begin{abstract}
Biochemical reference intervals are important for assessing the health of target species and populations by identifying abnormalities in key blood parameters. Although reference intervals have been established for baboons in captivity, the lack of data from free-ranging individuals makes it difficult to interpret the results of their blood chemistry panels or to assess and monitor the health of wild baboon populations. The goal of this study was therefore to establish serum biochemical reference intervals for free-ranging olive baboons (Papio anubis) in Kenya. We evaluated 14 biochemical parameters from 28 baboons sampled at the Mpala Research Center, Nanyuki, Kenya. Reference intervals obtained from this wild population were comparable to those from captive baboon populations. Alkaline phosphatase (ALP) and phosphorus levels differed significantly among age classes; both were higher in subadult and juvenile baboons than in adults. However, none of the components of the blood biochemistry panel differed significantly between the sexes. The reference intervals we report provide a baseline for the evaluation and treatment of free-ranging olive baboons and provide context for interpreting the biochemical profiles of captive individuals.
\end{abstract}

Keywords Chemical immobilization $\cdot$ Health status $\cdot$ Primate $\cdot$ Serum chemistry

\section{Introduction}

Well-defined, normal blood biochemistry parameters provide an important tool for assessing primate health at both the individual and population level. Because many

\author{
Margaret C. Crofoot \\ mccrofoot@ucdavis.edu \\ Jennifer C. Kishbaugh \\ KishbaughJ@si.edu
}

Extended author information available on the last page of the article 
factors can cause variation in blood biochemistry, including age, sex, and large-scale geographical location, samples from many individuals are needed to define the normal range of variation within a species. As a consequence of this need for large sample sizes, the reference intervals that veterinarians and researchers use to interpret the results of blood biochemistry tests are often based on data from captive, rather than wild, primate populations. However, components of blood chemistry panels may differ between captive and wild populations owing to a range of factors including diet, husbandry practices, and levels of physical activity and exertion and dehydration (Brenner et al. 2002; Junge and Louis 2005; Percin and Konyalioglu 2008; RangelMendoza et al. 2009). Such discrepancies, if they exist, could have a potentially important impact on our ability to assess and monitor the health of wild primates.

The olive baboon (Papio anubis) is one of two common, widespread baboon taxa in Kenya. Its close association with human settlements and agriculture make it a frequent patient of wildlife veterinarians. Further, the importance of olive baboons to public health in Kenya because of their role as potential carriers of zoonotic disease highlights a need for accurate reference values to assess baboon health. Although there are some compilations of normal blood biochemistry in captive baboons (Harewood et al. 1999; Teare 2002), few data exist from free-ranging individuals (Foy et al. 1965; Melton 1982), and no studies report the results of complete blood biochemistry panels for freeranging olive baboons. The goal of this study was to establish sets of reference values for common blood biochemistry test results from free-ranging olive baboons, taking variation due to age and sex differences into consideration.

\section{Methods}

\section{Study Location}

We conducted this research at the Mpala Research Center (MRC), located in central Kenya near the town of Nanyuki in Laikipia County. MRC covers 48,000 acres of unfenced savannah and woodland habitat. MRC is home to a diverse wildlife community that includes elephants, wild dogs, Grevy's and plains zebras, leopards, lions, and two nonhuman primate species: vervet monkeys and olive baboons. Olive baboons are numerous in the conservancy and surrounding settlements. Despite extensive shooting, trapping, and poisoning by humans, olive baboon populations in Kenya are on the rise and the species is listed as being of least concern (Kingdon et al. 2008).

\section{Study Design and Sample Collection}

We collected blood samples from 29 free-ranging olive baboons in July 2012 at the MRC as part of a larger study on baboon behaviour (Farine et al. 2017; StrandburgPeshkin et al. 2015, 2017). Only healthy individuals were selected for blood draws based on a brief visual exam and after being assessed as having body conditions scores of good (4/5; thin layer of subcutaneous fat, hip bones and spine are visible and readily palpable but not prominent) or excellent (5/5; well-developed musculature and subcutaneous fat layer, pelvis, ribs and spine were palpable with gentle pressure but not visible). We determined individual age and sex via physical examination, performed 
after the individual had been chemically immobilized. We estimated the age of each individual based on the pattern of dental eruption and evidence of sexual maturation. Individuals with deciduous dentition were classified as juveniles. Subadult and adult males were distinguished based on their body size and the development of secondary sexual characteristics, including their mantle, musculature, canine size, and morphology. We considered females to be adult if they had full, permanent dentition and were parous (based on the elongation and darkening of nipples) or showed evidence of cycling (based on the morphology of their sexual skin). Nulliparous females that were cycling but still had one or more deciduous teeth were classified as subadult.

For 1 mo before capture and sampling, we prebaited two open areas ca. $500 \mathrm{~m}$ apart with maize to habituate members of an olive baboon group to our traps. From July 21 to 29,2012 , we trapped 33 of the 46 members of this focal baboon group in $1 \times 1 \times 1 \mathrm{~m}$ cage traps. Four individuals deemed too small were immediately released. We immobilized the other 29 baboons using a $10-15 \mathrm{mg} / \mathrm{kg}$ dose of ketamine $\mathrm{HCl}$ (Ketamine $100^{\circledR}$, Pantex, Hapert, The Netherlands). We then weighed and measured these individuals, drew blood samples (3-4 ml, depending on body weight) and, except for two individuals, fit GPS collars (e-Obs Digital Telemetry, Gruenwald, Germany). The GPS collars were equipped with SureDrop automated breakaway units (Advanced Telemetry Systems, Isanti, MI, USA) that were preprogrammed to trigger on September 7, 2012, causing the collars to fall off. We successfully recovered all collars. We drew blood from the greater saphenous vein and placed it immediately into serum-separator tubes. We placed sample tubes in a darkened cooler with ice packs and centrifuged them to obtain serum as soon as we returned to the MRC labs (within a maximum of $9 \mathrm{~h}$ post-collection). It took ca. $15 \mathrm{~min}$ to complete the procedures for each baboon, after which we returned individuals to a cage trap to recover in the shade. All individuals were released without complications after 30$45 \mathrm{~min}$ and rejoined the rest of their group.

\section{Sample Analysis}

Serum chemistry analysis was performed with a VetScan VS2 ${ }^{\circledR}$ Analyzer (Abaxis Inc., Union City, CA, USA) using VetScan VS2 ${ }^{\circledR}$ Comprehensive Diagnostic Profile cassettes. The VetScan VS2 ${ }^{\circledR}$ is commonly used by veterinarians to process wildlife samples in the field, and despite a lack of published manufacturer's precision guidelines, it has shown acceptable reproducibility compared to central lab testing when analyzing nonhuman primate samples (Snider et al. 2009). Before field data collection, we also processed a serumbased control cassette to verify accuracy and ensure quality control while processing free-ranging baboon samples in the field. In total, 14 serum chemistry parameters were measured (albumin, alkaline phosphatase [ALP], alanine transaminase [ALT], amylase, total bilirubin, blood urea nitrogen [BUN], calcium, phosphorus, creatinine, glucose, sodium, potassium, total protein, globulin). The VetScan ${ }^{\circledR}$ analyzer recorded 26 of 28 sampled individuals as having high $(2+$ or $3+)$ levels of hemolysis. Hemolysis was severe enough in the samples from five individuals that the machine was unable to read potassium levels and, in one case, total bilirubin. Thus, samples from some baboons could not be used for all tests; the exact number of baboons contributing data to each chemistry parameter is listed in Table I. 


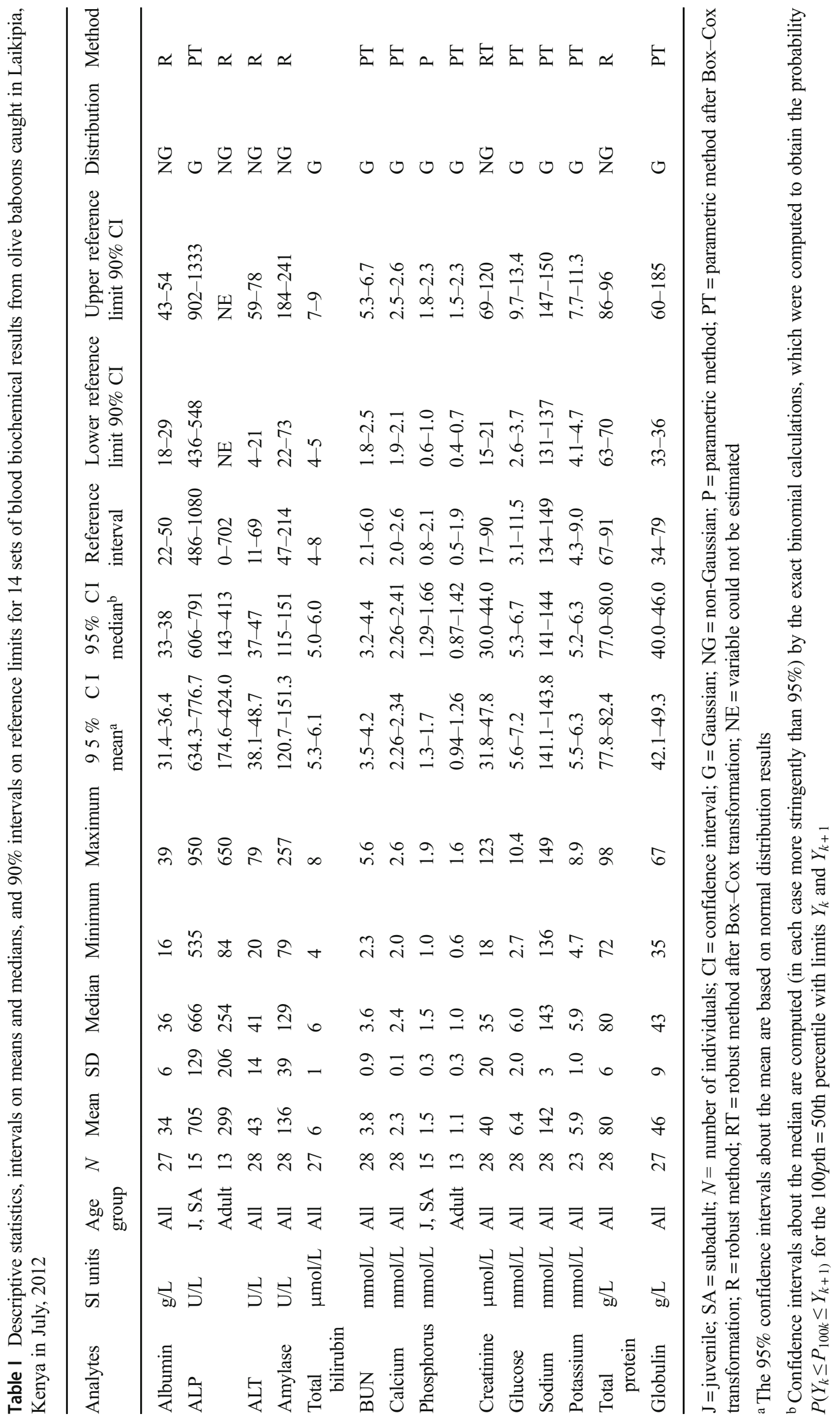




\section{Statistical Analysis}

We calculated descriptive statistics for each of the blood chemistry variables and determined the corresponding reference intervals using Reference Value Advisor (Geffré et al. 2011). All analyses conformed to CSLI guidelines; as the sample size was small $(N \leq 28)$ and the population variances were unknown, we averaged values and confidence intervals for each biochemical marker based on both the median and the mean because, in general, the median is less likely than the mean to be influenced by outliers and effects of skewed distributions in small sample sizes. The mean for each variable was estimated using the Student's $t$-distribution. Distribution-free confidence intervals were developed about each of the median values. We used the $k$ th order statistic, $Y_{k}$, which is a random variable, to develop these intervals for the median. The probability that our sample blood chemistry variables fall into the probability interval about the median is $P \geq 0.95$ with $N=29$, or less, depending on missing values and subsets of the data. We can assume each observation falls independently into or outside of this interval so that the desired probability is distributed as binomial $(N, p)$. These exact probabilities were calculated in Mathcad20 rather than using the normal approximation, despite some sample sizes being $>20$. General linear models were used to test for the effect of sex, age class, and their interaction on mean differences in biochemical markers. Levene's test for variances tested the assumption of homogeneity of variances. For the two variables, ALP and phosphorous, for which age category showed significant differences, separate reference confidence intervals were developed (Table I). One sample was excluded from analysis because of multiple obvious outliers and/or analyzer reading errors in the biochemistry results.

\section{Data Availability}

The datasets generated and analyzed in this study are available from the corresponding author.

\section{Ethical Note}

The Smithsonian Tropical Research Institute IACUC (assurance \#: 2012.0601.2015) approved all animal capture and treatment protocols. Protocols followed the guidelines prescribed by the Kenya Wildlife Service (KWS) Committee of the Department of Veterinary and Capture Services' guidelines on Wildlife Veterinary Practice (2006) and the Kenyan Veterinary Surgeons and Veterinary Para-Professionals Act (2011). The authors have no conflicts of interest to declare.

\section{Results}

Biochemical analyte interval values based on the mean vs. median were comparable in most cases (Table I). Three analytes - ALP, amylase, and creatinine - displayed more skewed distributions (average skew $=1.9$ ), with the median differing from the mean. Age had a significant influence on both ALP (adults $\bar{x}=299, \mathrm{SD}=206$; nonadults $\bar{x}=705$, $\mathrm{SD}=129 ; t(20)=-6.13, P<0.0001)$ and phosphorus (adults $\bar{x}=1.08, \mathrm{SD}=0.29$; 
nonadults $\bar{x}=1.45, \mathrm{SD}=0.20 ; t(26)=-3.35, P=0.002)$; juveniles and subadults exhibited higher levels of each than adults (see Table I). There was not a significant difference between juveniles and subadults for ALP (subadult $\bar{x}=688, \mathrm{SD}=119$; juvenile $\bar{x}=753$, $\mathrm{SD}=162 ; t(13)=0.85, P=0.41$ ) or phosphorus levels (subadult $\bar{x}=1.52, \mathrm{SD}=0.29$; juvenile $\bar{x}=1.28, \mathrm{SD}=0.22 ; t(13)=-1.49, P=0.16$ ). Age was not a significant factor for the remaining analytes and none of the analytes was influenced significantly by sex (see Table II). Moderate to severe hemolysis was present in 27 out of 28 samples.

Reference intervals and $90 \%$ confidence intervals on the reference limits were calculated for 14 serum biochemical analytes using Reference Value Advisor (Geffré et al. 2011; Table I). In most cases, our analyte interval values based on mean and median were comparable.

\section{Discussion}

In general, our reference intervals appeared similar to existing blood biochemistry parameters from the commonly used reference database Zoological Information Management System (https://zims.Species360.org; Table III), as well as from studies of other baboon species in captivity (Papio hamadryas: Harewood et al. 1999). However, these published reference intervals for captive individuals appeared to be based on multiple samplings from the same individuals and thus were not suitable for statistical comparison to either our mean or median based reference intervals, which were calculated from one sample per individual.

Only two biochemical analytes - ALP and phosphorus - differed significantly across age groups. Elevated levels of these analytes are frequently seen in juveniles and subadults, likely owing to increased bone growth and circulating growth hormone in growing individuals (Gilbert and Barresi 2016). Similar findings have been reported in other primate species (Fox et al. 2008; Havill et al. 2003) and in other mammal species more generally (García et al. 2010; Maas et al. 2013). Although current guidelines do not recommend partitioning reference intervals by age or sex class when sample sizes are less than 40 (Friedrichs et al. 2012), we provide separate reference intervals for adults and for subadults and juveniles. However, owing to the statistical challenges in accurately defining reference intervals with small sample sizes, the age group specific reference intervals calculated for ALP and phosphorus should be viewed with caution.

Creatinine has been positively correlated with muscle mass and incidence of glomerulonephropathy in older animals, as well as in animals that eat meat-rich diets (García et al. 2010). Our sample population was slightly skewed toward younger individuals, with 15 juveniles and subadults to 13 adults. Adult males, which generally have the greatest muscle mass and consequently higher creatinine levels, comprised only 3 of 28 individuals in our sample population. It is possible the younger age of our sample population and smaller percentage of adult males resulted in a lower range for creatinine. In addition, free-ranging baboons have a different diet from captive baboons. Although our sampled individuals were assessed as being clinically healthy, it is likely that their diet is less calorie- or protein-rich than captive populations, which may result in comparatively lower creatinine levels.

The frequency and severity of hemolysis in our samples could have influenced results across multiple analytes, particularly potassium levels. Poor venipuncture 


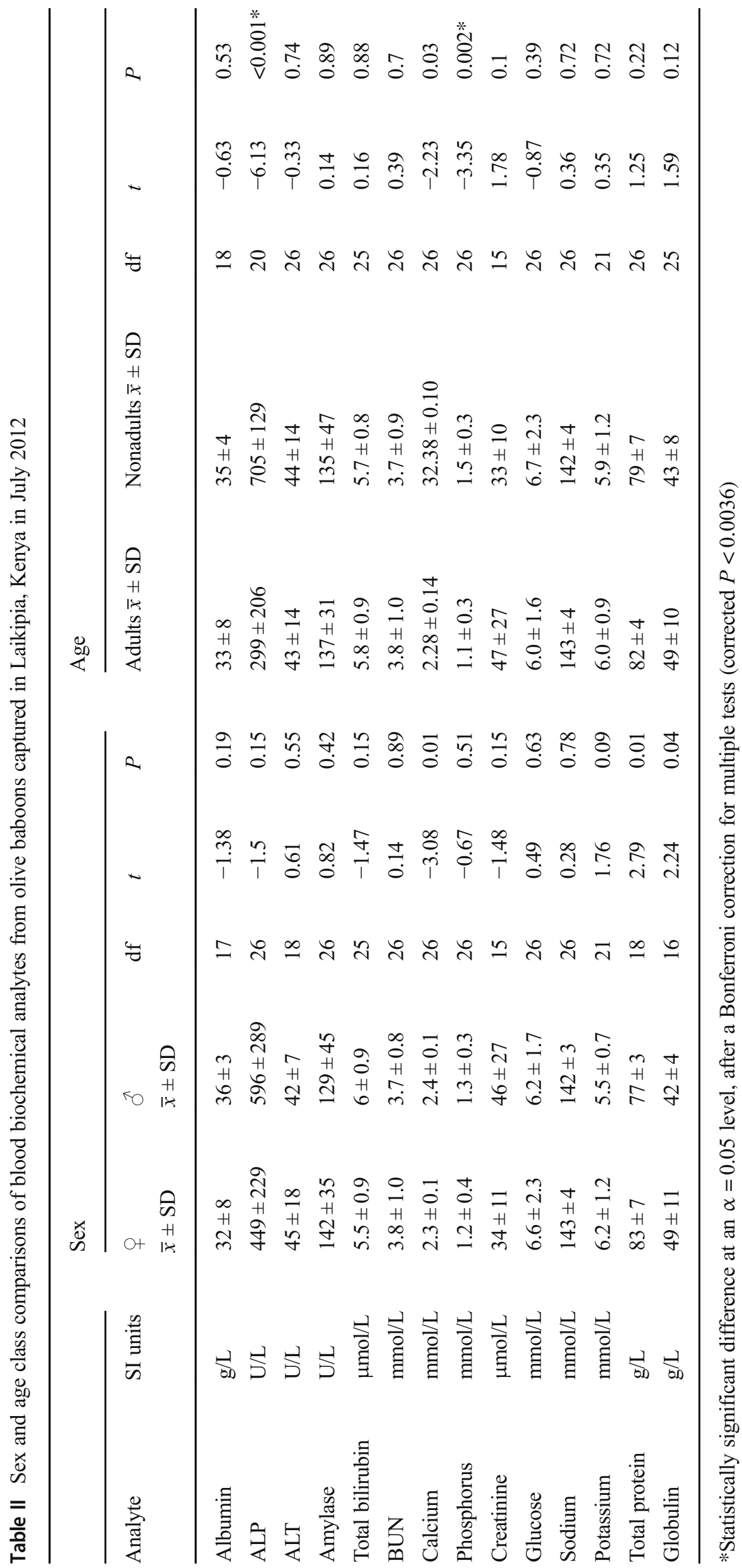


Table III Comparison of reference intervals for wild vs. captive baboons

\begin{tabular}{|c|c|c|c|c|}
\hline Analytes & $\begin{array}{l}\text { Wild P. anubis } \\
\text { RIs (this study) }\end{array}$ & $\begin{array}{l}\text { Captive } P \text {. } \\
\text { anubis RIs } \\
\left(\mathrm{ZIMS}^{\mathrm{a}}\right)\end{array}$ & $\begin{array}{l}\text { Captive }{ }^{\top} P \text {. } \\
\text { hamadryas Ris } \\
\text { (Harewood } \\
\text { et al. 1999) }\end{array}$ & $\begin{array}{l}\text { Captive }+P \text {. } \\
\text { hamadryas RIs } \\
\text { (Harewood } \\
\text { et al. } 1999 \text { ) }\end{array}$ \\
\hline Albumin $(\mathrm{g} / \mathrm{L})$ & $22-50$ & $29-45$ & $33-50$ & $32-50$ \\
\hline ALP (U/L) & $0-702$ & 69-1219 & $0-1485$ & $0-1400$ \\
\hline ALT (U/L) & $11-69$ & $21-55$ & $0-107$ & $0-122$ \\
\hline Amylase (U/L) & $47-214$ & N/A & N/A & N/A \\
\hline Total bilirubin $(\mu \mathrm{mol} / \mathrm{L})$ & $4-8$ & $0-6.84$ & $0-5.54$ & $0-4.08$ \\
\hline BUN (mmol/L) & $2.1-6.0$ & $2.29-8.43$ & N/A & N/A \\
\hline Calcium (mmol/L) & $2.0-2.6$ & $1.98-2.55$ & $2.04-2.68$ & $2.06-2.68$ \\
\hline Phosphorus (mmol/L) & $0.5-1.9$ & N/A & $\mathrm{N} / \mathrm{A}$ & N/A \\
\hline Creatinine $(\mu \mathrm{mol} / \mathrm{L})$ & $17-90$ & $44.2-123.6$ & $0-173.3$ & $34.1-93.8$ \\
\hline Glucose (mmol/L) & $3.1-11.5$ & $2.89-10.70$ & $2.58-7.97$ & $2.20-9.28$ \\
\hline Sodium (mmol/L) & $134-149$ & $135-158$ & $139-153$ & $138-153$ \\
\hline Potassium (mmol/L) & $4.3-9.0$ & $3.0-4.7$ & $2.65-4.75$ & $2.52-4.63$ \\
\hline Total protein $(\mathrm{g} / \mathrm{L})$ & $67-91$ & $53-81$ & $58-80$ & $59-84$ \\
\hline Globulin (g/L) & $34-79$ & $19-46$ & $\mathrm{~N} / \mathrm{A}$ & $\mathrm{N} / \mathrm{A}$ \\
\hline
\end{tabular}

a Zoological Information Management System (2018), http://zims.Species360.org/

technique, inadequate cold storage, and rough handling of samples can all cause damage to erythrocytes and lead to a hemolyzed sample in clinically healthy individuals (Ramer et al. 1995). In primates, serum potassium is elevated in the presence of even mild hemolysis due to high concentrations of intracellular potassium in erythrocytes (Ramer et al. 1995). Although proper sampling protocol was followed, the chaotic nature of collecting and transporting samples in the field likely led to a higher incidence of hemolysis in our samples than those in a more controlled captive setting and potentially also increased potassium levels. Given the likely increase in potassium due to hemolysis, the calculated reference intervals for potassium should be viewed with suspicion. There are no reference intervals of free-ranging olive baboons with which to compare creatinine or potassium. Therefore, it is also possible that these analytes are normally higher among free-ranging olive baboons.

In addition to the $90 \%$ CI obtained using Reference Value Advisor, we report $95 \%$ $\mathrm{CI}$ about both the sample means and medians. While the mean may be more useful in computing reference intervals for normally distributed data, the median is more appropriate for describing skewed distributions and less likely to be influenced by outliers. These effects are amplified by small sample sizes. Overall, however, the mean and median for each analyte were quite close and the distribution of values approximately symmetric. Thus, our computed reference intervals based about the mean were similar to those about the median for most analytes.

The analytes with more noticeable differences in these intervals included ALP, amylase, and creatinine. These analytes showed a more skewed distribution when compared to other tested analytes. Several analytes also had extreme outliers, most notably creatinine, for which one adult male sample had a value greater than four standard deviations higher 
than the mean. Both of these factors may make the median-based interval a more appropriate measurement of "typical" values in ALP, amylase, and creatinine for this species of wild baboon. Median-based intervals for the individual age groups for ALP and phosphorus are the most useful because they are exact for these small sample-sized groups when compared to the mean, although again these reference intervals should be viewed with caution owing to the overall low sample size. Ultimately, the decision of whether to use a mean or median-based approach should be made on a case-by-case basis, taking into consideration the factors that we described in the foregoing.

We conclude that wildlife veterinarians and researchers can use these reference intervals as a preliminary tool to assess the health of free-ranging olive baboons. However, only olive baboons from the Mpala Research Center were used in this study, and individuals from other regions in Kenya or Central Africa may differ in blood chemistry parameters. We propose that these values can also be a first approximation for captive olive baboons owing to the limited literature available. The Zoological Information Management System (ZIMS; http://zims.Species360.org/), a commonly used reference database for blood biochemistry information, for example, relies on samples of only 16-22 individuals for its reference intervals for this species (ZIMS Expected Test Results for Papio anubis [2018, August 2009]. Species360 Zoological Information Management System. Retrieved from http://zims.Species360.org) while captive studies with larger sample sizes (e.g., Harewood et al. 1999) were conducted on other baboon species. However, a comparative study based on one sample per individual is needed to recommend our values as a true reference for captive specimens.

Acknowledgments We thank Kenya National Science and Technology Council, the Kenyan Wildlife Service, and the Mpala Research Centre for permission to conduct this research (permit \#: NCST/RCD/ 12B/012/26B). We thank M. Wikelski, E. Bermingham, D. Rubenstein and M. Kinnaird for logistical support, and R. Kays, R. Lessnau, S. Alavi, J. Nairobi, R. Nelson, H. Nelson, M. Ngila, J. Halkano, and J. Kiseme for help with animal capture. We acknowledge funding from the NSF (IOS-EAGER-1250895; III-1514174), the Max Planck Institute for Ornithology, the Smithsonian Tropical Research Institute, and Princeton University. Dr. Joanna Setchell and two anonymous reviewers provided helpful feedback and suggestions on a previous version of this manuscript. M. C. Crofoot and S. Murray conceived this study. M. Mutinda, M. C. Crofoot, D. Zimmerman, and S. Murray conducted fieldwork and collected samples; S. Murray and D. Zimmerman analyzed samples. L. C. Hayek and J. C. Kishbaugh analyzed the data. D. A. Tunseth, M. Mutinda, D. Zimmerman, and M. C. Crofoot wrote the manuscript; all other authors provided editorial advice.

\section{References}

Brenner, D., Lewbart, G., Stebbins, M., \& Herman, D. W. (2002). Health survey of wild and captive bog turtles (Clemmys Muhlenbergii) in North Carolina and Virginia. Journal of Zoo and Wildlife Medicine, 33(4), 311-316.

Farine, D. R., Strandburg-Peshkin, A., Couzin, I. D., Berger-Wolf, T. Y., \& Crofoot, M. C. (2017). Individual variation in local interaction rules can explain emergent patterns of spatial organization in wild baboons. Proceedings of the Royal Society of London B: Biological Sciences, 284, 20162243.

Fox, M., Brieva, C., Moreno, C., MacWilliams, P., \& Thomas, C. (2008). Hematologic and serum biochemistry reference values in wild-caught white-footed tamarins (Saguinus leucopus) housed in captivity. Journal of Zoo and Wildlife Medicine, 39(4), 548-557.

Foy, H., Kondi, A., \& Mbaya, V. (1965). Hematologic and biochemical indices in the East African baboon. Blood, 26(5), 682-686. 
Friedrichs, K. R., Harr, K. E., Freeman, K. P., Szladovits, B., Walton, R. M., et al (2012). ASVCP reference interval guidelines: Determination of de novo reference intervals in veterinary species and other related topics. Veterinary Clinical Pathology, 41(4), 441-453.

García, I., Napp, S., Zorrilla, I., Vargas, A., Pastor, J., et al (2010). Determination of serum biochemical reference intervals for the Iberian lynx (Lynx pardinus). The Veterinary Journal, 183(2), 201-204.

Geffré, A., Concordet, D., Braun, J. P., \& Trumel, C. (2011). Reference value advisor: A new freeware set of macroinstructions to calculate reference intervals with Microsoft Excel. Veterinary Clinical Pathology, 40(1), 107-112.

Gilbert, S. F., \& Barresi, M. J. S. (2016). Developmental biology, 11th ed. Sunderland: Sinauer Associates.

Harewood, W. J., Gillin, A., Hennessy, A., Armistead, J., Horvath, J. S., \& Tiller, D. J. (1999). Biochemistry and haematology values for the baboon (Papio hamadryas): The effects of sex, growth, development and age. Journal of Medical Primatology, 28(1), 19-31.

Havill, L., Snider, C., Leland, M., Hubbard, G., Theriot, S., \& Mahaney, M. (2003). Hematology and blood biochemistry in infant baboons (Papio hamadryas). Journal of Medical Primatology, 32(3), 131-138.

Junge, R. E., \& Louis, E. E. (2005). Preliminary biomedical evaluation of wild ruffed lemurs (Varecia variegata and V. rubra). American Journal of Primatology, 66(1), 85-94.

Kingdon, J., Butynski, T. M., \& De Jong, Y. (2008). Papio anubis. IUCN Red List of Threatened Species.

Maas, M., Keet, D. F., \& Nielen, M. (2013). Hematologic and serum chemistry reference intervals for freeranging lions (Panthera leo). Research in Veterinary Science, 95(1), 266-268.

Melton, C. (1982). Blood parameters of the wild chacma baboon, Papio ursinus. African Zoology, 17(2), 85-90.

Percin, F., \& Konyalioglu, S. (2008). Serum biochemical profiles of captive and wild northern bluefin tuna (Thunnus thynnus L. 1758) in the Eastern Mediterranean. Aquaculture Research, 39(9), 945-953.

Ramer, J. C., MacWilliams, P., \& Paul-Murphy, J. (1995). Effects of hemolysis and frozen storage on serum electrolyte and chemistry values in cotton-top tamarins (Saguinus oedipus). Journal of Zoo and Wildife Medicine, 26, 61-66.

Rangel-Mendoza, J., Weber, M., Zenteno-Ruiz, C. E., López-Luna, M. A., \& Barba-Macías, E. (2009). Hematology and serum biochemistry comparison in wild and captive Central American river turtles (Dermatemys mawii) in Tabasco, Mexico. Research in Veterinary Science, 87(2), 313-318.

Snider, C., Dick Jr., E., McGlasson, D., Robbins, M., Sholund, R., et al (2009). Evaluation of four hematology and a chemistry portable benchtop analyzers using non-human primate blood. Journal of Medical Primatology, 38(6), 390-396.

Strandburg-Peshkin, A., Farine, D. R., Couzin, I. D., \& Crofoot, M. C. (2015). Shared decision-making drives collective movement in wild baboons. Science, 348(6241), 1358-1361.

Strandburg-Peshkin, A., Farine, D. R., Crofoot, M. C., \& Couzin, I. D. (2017). Habitat and social factors shape individual decisions and emergent group structure during baboon collective movement. ELife, 6, e19505.

Teare, J. (2002). Physiological data reference values. Eagan: International Species Information System.

\section{Affiliations}

\section{Mathew Mutinda ${ }^{1} \cdot$ Margaret C. Crofoot $^{2,3,4} \cdot$ Jennifer C. Kishbaugh ${ }^{5} \cdot$ Lee-Ann C. Hayek $^{6}$. Dawn Zimmerman ${ }^{5}$. Devin A. Tunseth ${ }^{5}$. Suzan Murray ${ }^{5}$}

1 Kenya Wildlife Service, Nairobi, Kenya

2 Department of Anthropology, University of California Davis, Davis, CA 95616-8522, USA

3 Animal Behavior Graduate Group, University of California Davis, Davis, CA 95616-8522, USA

4 Smithsonian Tropical Research Institute, Balboa, Ancon, Panama, Republic of Panama

5 Smithsonian Institution, National Zoological Park, Smithsonian Global Health Program, Washington, DC 20008, USA

6 Smithsonian Institution, National Museum of Natural History, Washington, DC 20013-7012, USA 